\title{
Neues zu Kopfschmerzen 2015 - ein Update
}

\section{What's New in Headache 2015? - an Update}

\author{
Autoren \\ M. Obermann ${ }^{1}$, D. Holle², S. Nägel ${ }^{2}$, J. Burmeister ${ }^{3}$, K. Solbach ${ }^{3}$, H.-C. Diener ${ }^{2}$ \\ Institute \\ ${ }^{1}$ Klinik für Neurologie und Westdeutsches Kopfschmerzzentrum, Universitätsklinikum Essen \\ ${ }^{2}$ Klinik für Neurologie, Universitätsklinikum Essen \\ ${ }^{3}$ Klinik und Poliklinik für Neurologie, Universitätsklinikum Essen
}

Schlüsselwörter
Kopfschmerzen
Migräne
O Spannungskopfschmerz
Medikamentenüberge-
brauchskopfschmerz
Trigeminusneuralgie
Keywords
headache
migraine
tension-type headache
medication overuse
headache
trigeminal neuralgia

Bibliografie

DOI http://dx.doi.org/

$10.1055 / \mathrm{s}-0035-1548943$

Akt Neurol 2015; 42: 247-255

(c) Georg Thieme Verlag KG

Stuttgart · New York

ISSN 0302-4350

\section{Korrespondenzadresse}

\section{Dr. Mark Obermann}

Klinik für Neurologie und Westdeutsches

Kopfschmerzzentrum

Universitätsklinikum Essen

Hufelandstraße 55

45122 Essen

Mark.Obermann@uk-essen.de

\section{Zusammenfassung \\ $\nabla$}

Die neuesten und relevanten Informationen über Kopf- und Gesichtsschmerzen werden hier kurz und übersichtlich dargestellt. Eine Studie aus den USA konnte den Wert einer guten Anamnese und eines einfühlsamen, informativen Patientengespräches noch einmal unterstreichen. Gerade in der Schmerztherapie spielt eine gute und fundierte Aufklärung der Patienten über die geplante Therapie eine herausragende Rolle. Neue medikamentöse Therapieoptionen zur Behandlung der Migräne mit monoklonalen Antikörpern gegen den calcitonin-gene related peptide (CGRP) Rezeptor oder CGRP selbst haben die Phase-2-Studien erfolgreich beendet und machen nun einen weiteren Schritt in Richtung Patientenversorgung. Ein weiteres neues Medikament zur Behandlung der Trigeminusneuralgie hat die Phase-2-Studie erfolgreich beendet. Unterschiedliche Verfahren der Neuromodulation sind ebenfalls weiter „en vogue“ und werden in klinischen Studien, evidenzbasiert getestet. Hier ist die transkutane Stimulation des Nervus vagus besonders hervor zu heben, die sich in den folgenden Phase-3-Studien als einfach zu handhabende und wirksame Therapie erweisen könnte. Neben der Therapie werden auch neue pathophysiologische und klinisch-differenzialdiagnostische Erkenntnisse bei unterschiedlichen Kopfschmerzerkrankungen vorgestellt.

\section{Einleitung}

Diese Übersicht fasst die neuesten Forschungsarbeiten aus dem erweiterten vergangenen Jahr von November 2013 bis Februar 2015 zusammen und konzentriert sich hierbei auf die Arbeiten die aktuell oder potentiell unser Verständnis der Diagnose und Therapie unterschiedlichen Kopfschmerzerkrankungen verändern könnten. Das Forschungsumfeld im Bereich Kopf- und Gesichts-

\section{Abstract \\ $\nabla$}

A systematic review of the latest and most relevant information on different disorders of head and facial pain is presented. A study from the United States has highlighted the value of good history taking and a sensitive and informative patient dialogue. Especially in pain therapy, thorough patient education about the planned treatment approach plays a prominent role. New drug therapies for the treatment of migraine with monoclonal antibodies against the calcitonin generelated peptide (CGRP) receptor or the CGRP itself showed promising results in the successfully completed phase-II-studies and represent a further step in patient care. A new drug for the treatment of trigeminal neuralgia has also successfully completed phase-II-trial. Different methods of neuromodulation remain "en vogue" and will be tested in evidence-based, clinical trials. Transcutaneous stimulation of the vagus nerve (tVNS) demonstrated its efficacy in cluster headache and migraine in early phase-II-studies and could prove to be an easy-to-use and effective therapy in phase-III-clinical trials. In addition to therapy options, new pathophysiological and differential diagnostic findings in different primary headache disorders are presented.

schmerzen ist weiterhin sehr aktiv und die Bemühungen um ein besseres Verständnis der Pathophysiologie, Epidemiologie und Therapie werden auf einem hohen Forschungsniveau von verschiedenen Arbeitsgruppen in Europa, Südostasien und Nordamerika vorangetrieben. Die neue und dritte Fassung der Kopfschmerzklassifikation (ICHD-III beta) der Internationalen Kopfschmerzgesellschaft (IHS) hat viele klinische Forschungsgruppen dazu ermuntert die hier vorge- 
schlagenen Diagnosekriterien noch einmal zu überprüfen und im wirklichen Leben zu testen. Diese „Feldversuche“ im klinischen Alltag sind für die alltägliche Einschätzung der unterschiedlichen Patientenbeschwerden sehr wichtig und betreffen nicht nur die seltenen, eher unbekannten Kopfschmerzentitäten, sondern auch die sehr häufigen primären Kopfschmerzen wie Migräne oder Spannungskopfschmerz.

Im letzten Jahr rückten neue Therapieoptionen der Migräneprophylaxe in den Fokus des wissenschaftlichen Interesses. Monoklonale Antikörper gegen Calcitonin gene-related peptide (CGRP) und dessen Rezeptor zeigten sehr vielversprechende Ergebnisse in Phase-2-Studien. Die Entwicklung von oralen CGRP Antagonisten wird allerdings aufgrund von Unverträglichkeiten und Nebenwirkungen von den meisten Firmen nicht weiter fortgeführt. Darüber hinaus ist die Begeisterung für unterschiedliche Verfahren der Neuromodulation ungebrochen und es wurden einige gut gemachte Studien in diesem Zusammenhang publiziert. Auch bei den selteneren Kopf- und Gesichtsschmerzformen kündigen sich neue, innovative Therapieoptionen an. Die Phase2-Studie eines neuen selektiven Natrium-Kanal-Blockers zeigte eine gute Wirksamkeit in der Therapie der Trigeminusneuralgie. Auch die Behandlung der benignen intrakraniellen Hypertension (Pseudotumor cerebri) mit Acetazolamid konnte erstmals wissenschaftlich belegt werden. Beim Cluster Kopfschmerz wurde die Rolle des Hypothalamus für die Pathophysiologie erneut in Frage gestellt - eine Veränderung der grauen Substanz in diesem Bereich konnte nicht nachgewiesen werden.

\section{Epidemiologie}

$\nabla$

Auch im letzten Jahr wurden die Ergebnisse zahlreicher großer populationsbasierter Studien veröffentlicht und insbesondere die Zusammenhänge zwischen Kopfschmerzen und Stress, sowie psychiatrischen Komorbiditäten untersucht.

In einer US-amerikanischen Publikation wurden systematisch aus 4 großen populationsbasierte Datenbanken (National Health Interview Survey, National Ambulatory Medical Care Survey National Hospital Ambulatory Health Care Survey und Defense Medical Surveillance System) aktualisierte epidemiologische und demografisches Daten zur Migräne und anderen schweren Kopfschmerzerkrankungen erhoben, welche ein ziemlich akkurates Bild der amerikanischen Gesamtbevölkerung widerspiegeln [1]: Es ergab sich eine 3-Monats-Prävalenz für Migräne von 14,2\% (Frauen 19,1\%, Männer 9,0\%) mit im Verlauf unveränderten Prävalenzraten zwischen 2005 und 2012. Kopfschmerzerkrankungen bleiben weiterhin ein gewichtiges Problem in der Gesundheitsversorgung und stellten im Zeitraum zwischen 2009 und 2010 den insgesamt vierthäufigsten Konsultationsgrund $(3,1 \%$ aller Patientenkontakte) in der Notaufnahme dar. Eine longitudinale, populationsbasierte Studie aus Deutschland untersuchte den Zusammenhang zwischen subjektivem Stresserleben und Kopfschmerzhäufigkeit [2]. Hierbei erhielten Studienteilnehmer über einen Zeitraum von 2 Jahren alle 3 Monate einen Fragebogen, in welchem das subjektive Stresserleben in einer modifizierten visuellen Analogskala von 0 bis 100 erhoben wurde. Von den 5159 Teilnehmern, die min. einen Fragebogen beantworteten litten 31\% unter Kopfschmerz vom Spannungstyp (TTH) und 14\% unter Migräne. Es konnte bei Patienten mit TTH ein Zusammenhang zwischen einer VAS-Erhöhung um 10 Punkte und einer Kopfschmerzhäufung um 6\%. Bei Migränepatienten betrug dieser Effekt 4,3\%.

\section{Risikofaktoren und Komorbiditäten \\ $\nabla$}

Die Komorbidität von Kopfschmerz und psychiatrischen Syndromen ist ein bekanntes klinisches Phänomen. Eine weltweit durchgeführte Untersuchung im Rahmen des WHO World Mental Health Survey an 52095 Teilnehmern konnte eine Korrelation von affektiven Störungen, Angsterkrankungen und Störungen der Impulskontrolle und späterem Auftreten von häufigen und schweren Kopfschmerzen nachweisen [3].

Die in Norwegen durchgeführte populationsbasierte YoungHUNT-Studie erfasste Depressions- und Angst-Scores bei Heranwachsenden im Alter von 12 bis 17 Jahren und beinhaltete ein Follow-up nach 4 Jahren. Es konnte ein statistisch signifikanter Zusammenhang von höheren Depressions- und Angst-Scores bei Baseline und erhöhtem Auftreten häufiger Kopfschmerzen im Follow-up nachgewiesen werden. Dieser Zusammenhang bestand auch für die Subgruppen Migräne und nicht klassifizierbarer Kopfschmerz, jedoch nicht für Kopfschmerzen vom Spannungstyp [4].

Ein weiterer wichtiger Faktor, der zunehmend in den wissenschaftlichen Fokus rückt, ist der Zusammenhang zwischen Kopfschmerzen und Ernährung: Eine Querschnittsuntersuchung im Rahmen der Women's Health Study erfasste das Vorliegen migränöser und nicht-migränöser Kopfschmerzen und semiquantitativ die Einnahme von Lebensmitteln, welche möglicherweise zu einer Kopfschmerzhäufung führen können [5]. Bei der Women's Health Study handelt es sich um eine große US-amerikanische Studie zur Untersuchung präventiver Effekte von Vitamin E und Azetylsalizylsäure an insgesamt 39876 weiblichen Beschäftigten im Gesundheitssektor. 25755 Frauen litten unter Migräne und 5573 unter nicht-migränösen Kopfschmerzen. Beide Gruppen waren mit einem niedrigen Alkoholkonsum assoziiert, möglicherweise als Ausdruck eines Vermeidungsverhaltens. Migräne-Patientinnen mit Aura gaben einen niedrigeren Konsum von Schokolade, Eiscreme, Hot Dogs und anderen Fleischprodukten an, während bei Patientinnen mit einer Migränefrequenz von > 1/Woche ein Zusammenhang mit niedrigem Wein- und Milchkonsum besteht. Die Unterschiede zu Teilnehmerinnen ohne Kopfschmerzen bzw. zu Teilnehmerinnen mit nicht migräniformen Kopfschmerzen werden von den Autoren als mögliches migränespezifisches Ernährungsprofil gedeutet, welches infolge zuvor getriggerter Attacken bestehen könnte.

Eine weitere, US-amerikanische Querschnittsstudie befasste sich mit dem Zusammenhang von Kopfschmerzen bei simultaner Einnahme von Statinen und der Serumkonzentration von 25-Hydroxy-Vitamin D [6]. Während es für Statine Hinweise auf eine mögliche migräneprophylaktische Wirkung gibt, ist bezüglich Vitamin D lediglich ein Zusammenhang zwischen niedriger Blutkonzentration und häufigen Kopfschmerzen beschrieben. Die multivariate Regressionsanalyse der 5938 Studienteilnehmer ergab einen signifikanten Zusammenhang zwischen hohen 25-Hydroxy-Vitamin-D-Serumkonzenttationen(d.h. > $57 \mathrm{nmol} / \mathrm{L}$ ) bei simultaner Statin-Einnahme und niedrigen Prävalenzen für Migräne und anderen schweren Kopfschmerzerkrankungen.

Ein häufig diskutierter Risikofaktor für die Chronifizierung von Migräne ist das unzureichende Ansprechen auf Akuttherapeutika. Dieser ist nun im Rahmen der American Migraine Prevalence and Prevention Study näher untersucht worden [7]. Hierbei wurde ein validierter Fragebogen (Migraine Treatment Optimisation Questionnaire) eingesetzt und das Therapieansprechen in 4 Kategorien eingeteilt. Von den 5681 Teilnehmern mit episodischer Migräne entwickelten insgesamt 3,1\% nach einem Jahr 
eine chronische Migräne. Die Rate einer neu diagnostizierten chronischen Migräne betrug nur 1,9\% in der Gruppe mit initial höchstem Therapieansprechen im Vergleich $\mathrm{zu}$ 6,8\% in der Gruppe mit niedrigstem Ansprechen. Es wurde ein an die möglichen Störfaktoren Kopfschmerzhäufigkeit und Grad der Einschränkung durch Kopfschmerz („disability“) adjustiertes Modell erstellt. In diesem hatte weiterhin die Gruppe mit dem niedrigsten Therapieansprechen ein mehr als doppelt so hohes Risiko für eine Migränechronifizierung als die Gruppe mit dem besten Therapieansprechen. Zu klären bleibt jedoch weiterhin die Kausalität dieses Zusammenhangs und damit die Frage, ob Patienten mit hoher Kopfschmerzhäufigkeit aufgrund der Schwere der Erkrankung ein schlechtes Ansprechen auf die Akutbehandlung zeigen oder ob eine unzureichende Wirksamkeit der Akuttherapie chronische Krankheitsverläufe begünstigt.

\section{Akuttherapie der Migräne \\ $\nabla$}

Im heutigen medizinischen System kommt der sprechenden Medizin eine immer kleinere Rolle zu, da dieser Aspekt finanziell nur unzureichend abgebildet ist. Gerade in der Schmerzmedizin spielt die Aufklärung des Patienten über die Erkrankung sowie die einzunehmenden Medikamente aber eine wichtige Rolle und beeinflusst den Behandlungserfolg maßgeblich. Wells et al. konnten diesen klinischen Eindruck nun auch mittels einer fragebogenbasierten Studie in 7 überregionalen Kopfschmerzzentren in den USA belegen [8]. Dabei zeigte sich, dass nur wenige Minuten mehr Aufklärung über die zu erwartenden Wirkung sowie Nebenwirkungen der Migräneakutmedikation zu einer deutlichen besseren Compliance der Medikamenteneinnahme führten und damit den Behandlungserfolg signifikant verbesserten. Auf Dauer könnte mit einer ausführlicheren Information des Patienten sicherlich mehr Geld gespart werden (z. B. durch Vermeidung der Entstehung eines Kopfschmerzes bei Medikamentenübergebrauch mit entsprechenden Folgekosten), als man mit einer initial unzureichenden kurzen Aufklärung einspart!

Neuromodulative Verfahren erfahren einen immer höheren Stellenwert in der Behandlung von Kopfschmerzerkrankungen, insbesondere der Migräne. Dabei spielen vor allem nicht-invasive Behandlungsformen eine wichtige Rolle, da diese mit deutlich weniger Nebenwirkungen und Risiken verglichen mit invasiven neuromodulativen Verfahren verbunden sind. Diese Verfahren könnten in Zukunft eine Behandlungsoption bei denjenigen Patienten darstellen, die Triptane nicht vertragen oder aufgrund von Nicht-Wirksamkeit oder bestehender Komorbiditäten nicht einnehmen. In einer ersten offenen Studie wurde die Behandlung akuter Migräneattacken mittels nicht-invasiver Stimulation des Nervus vagus untersucht [9]. In dieser Studie wurden insgesamt 80 Migräneattacken bei 30 Patienten behandelt. Dabei zeigte sich bei etwa einem Fünftel der Patienten eine Schmerzfreiheit nach 2 Stunden, bei mehr als jedem dritten Patienten fand sich eine Besserung der Beschwerden nach diesem Zeitraum. Es traten lediglich leichte Nebenwirkungen in Form von Verspannungen im Nacken sowie Schmerzen im Stimulationsgebiet am Hals auf. Die Wirksamkeit des Verfahrens wird sich allerdings erst nach der Durchführung plazebo-kontrollierter Studien wirklich beurteilen lassen, da bei neuromodulativen Verfahren dieser Art mit einem hohen Plazebo-Effekt zu rechnen ist. Große kontrollierte Studien zum Einsatz dieses Verfahrens sind für das Jahr 2015 geplant. Kritisch muss zu diesem Verfahren jedoch angemerkt werden, dass bisher der Nachweis aussteht, dass es sich um eine spezifische Wirkung der Vagus-Stimulation handelt bzw. der Nervus vagus überhaupt stimuliert wird.

Auch viele Kinder und Jugendliche leiden unter Migräne, wobei sich die Frage nach der besten Akutmedikation stellt. In einer multizentrischen Plazebo-kontrollierten randomisierten Studie in Japan wurde nun die Wirksamkeit von Sumatriptan 25 und $50 \mathrm{mg}$ bei insgesamt 144 Migräneattacken untersucht [10]. Hierbei zeigte sich keine klinisch relevante Wirksamkeit des Triptans verglichen mit Plazebo. Diese Studie belegt erneut die Schwierigkeit von Studien zur Akutmedikation im Kindesalter, da sich (trotz vielversprechender Wirksamkeit des Verums) ein so hoher Plazebo-Effekt findet, dass sich keine Wirksamkeit des Präparates belegen lässt. Zudem ist die in dieser Studie eingesetzte Dosierung des Sumatriptans relativ niedrig (auch wenn sie der üblichen Dosierung im Kindesalter entspricht), was auch zur schwachen Wirksamkeit des Präparates beigetragen haben könnte. Inwieweit möglicherweise auch eine Unreife des kindlichen Gehirns für das mangelnde Ansprechen des Triptans verantwortlich sein könnte, ist bisher nicht ausreichend untersucht, Basierend auf der vorliegenden Datenlage, erscheint der Einsatz von Triptanen in Einzelfällen gerechtfertigt, wenn andere nichtmedikamentöse und medikamentöse Therapieoptionen keinen ausreichender Therapieerfolg gebracht haben [11].

Bei Erwachsenen ist die Wirksamkeit der Triptane dagegen eindeutig belegt. Aktuell stehen zur Akuttherapie der Migräne 7 Präparate zur Verfügung. In einer großen Metaanalyse wurde nun die Wirksamkeit der einzelnen Triptane miteinander verglichen [10]. Dabei zeigten Eletriptan, Rizatriptan sowie Frovatriptan den größten therapeutischen Effekt. Allerdings muss für den einzelnen Patienten natürlich individuell geschaut werden, welches der Triptane am besten wirksam und mit den wenigsten Nebenwirkungen vergesellschaftet ist.

\section{Prophylaxe der Migräne}

Für viele der zugelassenen Migräneprophylaktika wie Betablocker, Flunarizin, Antikonvulsiva oder trizyklische Antidepressiva besteht das Problem der Patienten-Compliance bedingt durch die häufigen Nebenwirkungen. Am schwierigsten ist hier die Gewichtszunahme, die mit allen Migräneprophylaktika außer Topiramat auftritt. Daher ist es wichtig, neue Substanzen zu entwickeln, die diese Nebenwirkungen nicht haben.

Ein potentiell neues Medikament zur Migräneprophylaxe ist das Antihypertensivum Candesartan. Im Jahr 2003 hatte bereits eine Arbeitsgruppe in Norwegen die prophylaktische Wirkung von Candesartan in einer kleinen Studie bei der Migräne belegt [12]. Dieselbe Arbeitsgruppe hat jetzt eine weitere Plazebo-kontrollierte doppelblinde Cross over-Studie durchgeführt [13]. Die Patienten wurden entweder mit $16 \mathrm{mg}$ Candesartan, $160 \mathrm{mg}$ retardiertem Propranolol oder Plazebo behandelt. Es erfolgte eine jeweils 12-wöchige Behandlungsphase gefolgt von einer 4-wöchigen Auswaschphase. Eingeschlossen wurden Patienten mit Migräne mit und ohne Aura, die mindestens 2 Migräneattacken pro Monat aufwiesen. Der primäre Endpunkt der Studie waren Tage mit mittelschweren oder schweren Kopfschmerzen, die mindestens 4 Stunden anhielten oder die mit spezifischen Migränemitteln behandelt wurden. Initial wurden 72 Patienten in die Studie eingeschlossen und randomisiert. Für die abschließende ITT-Analyse standen die Ergebnisse von 61 Patienten zur Verfügung. Die meisten Patienten waren weiblich (83\%) und das mittlere Alter betrug 37 Jahre. Die mittlere Frequenz der Migrä- 
neattacken pro Monat betrug 4,8. Für Plazebo ergab sich eine Reduktion der Zahl der Migräneattacken pro Monat von 4,82 in der Baseline auf 3,53. Die Reduktion gegenüber der Baseline betrug unter Candesartan 2,95 und unter Propranolol 2,91. Statistisch gesehen waren beide aktiven Behandlungsgruppe signifikant besser wirksam als Plazebo. Zwischen Candesartan und Propranolol bestand statistisch kein Unterschied. Die meisten sekundären Endpunkte waren für Candesartan und Propranolol ebenfalls gegenüber Plazebo überlegen. Die Zahl der Responder mit einer mindestens 50\%-igen Reduktion der Migränetage pro Monat betrug 23\% für Plazebo, $43 \%$ für Candesartan und $40 \%$ für Propranolol. Unter der relativ hohen Dosis von $16 \mathrm{mg}$ Candesartan waren die am häufigsten geklagten Nebenwirkungen Schwindel, allgemeine Schmerzen, Schlafstörungen und Müdigkeit. Unter $160 \mathrm{mg}$ Propranolol traten diffuse Körperschmerzen, unsystematischer Schwindel, Schlafstörungen und Müdigkeit auf. Es gibt jetzt 2 zwar relativ kleine aber gut durchgeführte Studien zur Wirksamkeit von Candesartan in der Prophylaxe der Migräne. Obwohl die Substanz für diese Indikation nicht zugelassen ist, ist es angesichts der relativ geringen Nebenwirkungen vertretbar, bei Patienten, die die traditionellen Migräneprophylaktika nicht vertragen, einen Therapieversuch zu unternehmen. Allerdings wären weitere Studien mit größeren Patientenzahlen wünschenswert.

Calcitonin Gene Related Peptide (CGRP) spielt eine wichtige Rolle bei der Pathophysiologie der Migräne [14]. In den letzten Jahren war eine Reihe von CGRP-Antagonisten zur Behandlung akuter Migräneattacken entwickelt worden. Diese Substanzen waren wirksam. Allerdings wurde die Entwicklung von CGRP-Antagonisten eingestellt, nachdem einige dieser Substanzen bei regelmäßiger Einnahme zu Leberschäden führten [15].

Ein weiterer Ansatz ist jetzt die Entwicklung von humanisierten monoklonalen Antikörpern entweder gegen CGRP selbst oder gegen den CGRP-Rezeptor. Für 2 der neuen monoklonalen Antikörper gegen CGRP gibt es jetzt Phase-II-Resultate. ALD403 ist ein humanisierter monoklonaler Antikörper gegen CGRP, der eine lange Halbwertzeit von 31 Tagen hat. Der Antikörper kann die Bluthirnschranke nicht überwinden und sollte daher keine zentralen Nebenwirkungen haben. In einer Phase-II-Studie wurden Migränepatienten im Alter zwischen 18 und 55 Jahren eingeschlossen [16]. Ein weiteres Einschlusskriterium war die Zahl der Migränetage pro Monat, die zwischen 5 und 14 Tagen liegen mussten. Die Patienten erhielten über einen Zeitraum von 12 Wochen alle 2 Wochen eine Infusion. Diese enthielt entweder 1000 mg ALD403 oder Plazebo. Primärer Endpunkt war die Häufigkeit der Migränetage in den Wochen 5-8 im Vergleich zur Baseline. Die Studie randomisierte 82 Patienten in die PlazeboGruppe und 81 Patientin in die Verum-Gruppe. Die Patienten waren überwiegend weiblich $(80 \%)$ und das mittlere Alter betrug 39 Jahre. Die durchschnittliche Zahl der Migräneattacken pro 28 Tage in der Baseline betrug 8,5 und die Zahl der Migräneattacken lag zwischen 6 und 6,7. Da die Studie in erster Linie eine Sicherheitsstudie war, wurden zunächst die Nebenwirkungen berichtet. Die Nebenwirkungen waren gering und unter ALD403 kam es Husten, Schnupfen, Heiserkeit und Harnwegsinfektionen. Alle anderen Nebenwirkungen waren gleich häufig wie in der Plazebo-Gruppe. Bei den Laborwerten ergaben sich keine Auffälligkeiten. Die Reduktion der Migränetage in den Wochen 5-8 verglichen mit der Baseline betrug -5,6 Tage für ALD403 und -4,6 Tage für die Plazebo-Gruppe. Dieser Unterschied war statistisch signifikant. Diese relativ kleine Studie zeigt, dass der CGRP-Antikörper offenbar wirksam ist. Er ist auch gut verträglich. Die absolute Differenz von einem Migränetag pro Monat ist allerdings relativ gering. Jetzt müssen größere Phase-III-Studien zeigen, ob sich der Therapieeffekt bei größeren Patientenpopulationen reproduzieren lässt.

LY2951742 ist ein weiterer monoklonaler humanisierter CGRPAntikörper. In einer Phase-II-Studie bei häufiger episodischer Migräne wurde die Substanz alle 2 Wochen in einer Dosis von $150 \mathrm{mg}$ subkutan appliziert [17]. Der primäre Endpunkt der Studie war eine Reduktion der Migränetage in den Wochen 9-12 der 12-wöchigen Behandlungsphase. Unter Verum kam es zu einer Reduktion der Migränetage um 4,2 Tage und unter Plazebo um - 3 Tage. Es traten keine relevanten Nebenwirkungen auf.

Ein weiterer monoklonaler Antikörper ist LBR-101, der jetzt in Phase-II-Studien untersucht wird [18].

Repetitive Blockaden des N. occipitalis major mit Corticosteroiden und/oder Lokalanästhetika sind in der Kurzzeitprophylaxe des Cluster-Kopfschmerzes wirksam. Diese Technik wurde nun zur Kurzzeitprophylaxe der Migräne untersucht [19]. In die Studie wurden Patienten mit häufigen Migräneattacken oder chro-

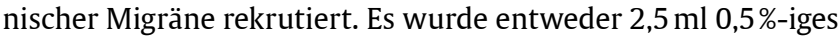
Bupivacain+0,5 ml Methylprednisolon entsprechend $20 \mathrm{mg}$ oder Kochsalzlösung mit 1\%-igem Lidocain injiziert. Die Patienten führten einen Monat vor und nach der Injektionsserie ein Kopfschmerz-Tagebuch. Der primäre Endpunkt war eine mindestens 50\%-ige Reduktion der Kopfschmerztage. 34 Patienten erhielten die Verum- und 35 Patienten die Plazebo-Behandlung. Die Patienten hatten zur Baseline pro Monat 9 Tage mit mittelschweren und 4 Tage mit schweren Kopfschmerzen. Die Zahl der Responder betrug in beiden Therapiegruppen 30\% und der Unterschied zwischen den beiden Therapiegruppen war statistisch nicht signifikant. Daher eignet sich diese Methode nicht zur Kurzzeitprophylaxe bei Patienten mit häufigen Migräneattacken.

Die hochfrequente repetitive transcranielle Magnetstimulation wird bereits zur Behandlung von chronischen neuropathischen Schmerzen und Phantomschmerzen eingesetzt. In einer randomisierten Plazebo-kontrollierten Studie wurde dieses Verfahren jetzt zur Prophylaxe der chronischen Migräne untersucht [20]. In die Studie wurden 18 Patientinnen mit chronischer Migräne eingeschlossen. Die Einnahme von Migräneprophylaktika war untersagt. Es wurden über einen Zeitraum von 8 Wochen 23 Sitzungen mit hochfrequenter transcranieller Magnetstimulation über dem dorsolateralen präfrontalen Cortex durchgeführt. Die Studie war negativ. Das Ergebnis ist allerdings bei einer relativ geringen Patientenzahl mit einer schwierig zu behandelnden Erkrankung auch nicht verwunderlich.

Unter den Migräneprophylaktika wird weiterhin Valproinsäure eingesetzt. Angesichts der teratogenen Wirkung sollte der Einsatz von Valproinsäure allerdings extrem zurückhaltend bei Frauen im gebärfähigen Alter erfolgen. Die Indikation besteht nur, wenn andere Migräneprophylaktika nicht wirksam waren, nicht vertragen wurden oder kontraindiziert sind. Mit der Verschreibung muss eine ausführliche Aufklärung bezüglich Antikonzeption und dem Risiko von schwerwiegenden Missbildungen erfolgen.

\section{Kopfschmerz bei Medikamentenübergebrauch (MOH, MÜKS) \\ $\nabla$}

Lange ist bekannt, dass die häufige Einnahme von Schmerz- oder Migränemittel bei vorbestehender primärer Kopfschmerzerkrankung zur Entwicklung eines Kopfschmerz bei Medikamenten- 
übergebrauch $(\mathrm{MOH}) /$ Medikamentenübergebrauchkopfschmerz (MÜKS)) führen kann. Weniger ist allerdings über die Zusammenhänge des MÜKS mit psychiatrischen Komorbiditäten bekannt. Eine große offene prospektive multinationale Multizenterstudie des EU geförderten COMOESTAS Projektes untersuchte genau diese Zusammenhänge und konnte eindrucksvoll zeigen, dass die Beendigung des Übergebrauchs nicht nur die Kopfschmerzsymptomatik, sondern parallel auch die psychiatrischen Komorbiditäten positiv beeinflussen kann [21]. In die Studie wurden insgesamt 694 Patienten mit Übergebrauchskopfschmerz eingeschlossen. Von 492 dieser Patienten lagen prospektive 6 Monatsdaten vor. $80 \%$ der Patienten waren weiblich, die meisten litten primär unter einer Migräne. Die durchschnittliche Anzahl der Kopfschmerztage betrug 24 pro Monat. Chronischer Kopfschmerz bestand im Mittel seit 5-6 Jahren. Am häufigsten wurde Analgetika-, folgend Kombinationsanalgetika- und Triptan-Übergebrauch beobachtet. Durch Medikamentenentzug und Initiierung einer Migräne-Prophylaxe konnten die Kopfschmerztage um $60 \%$ auf durchschnittlich 10 Tage reduziert werden. Die Einschränkung der Lebensqualität auf der MIDAS (Migraine Disability Assessment)-Skala verbesserte sich um 57\%. Die Zahl der Patienten mit einer Depression konnte um 50\% reduziert werden. Bezüglich Angsterkrankungen konnte immerhin eine Reduktion von 284 auf 207 (27\%) erreicht werden. Der Zusammenhang von Migräne und psychiatrischen Komorbiditäten, insbesondere der wechselseitige Einfluss aufeinander, konnte damit erstmals an einer großen, wenn auch nicht kontrollierten Stichprobe gezeigt werden.

Eine neuerliche Analyse der großen populationsbezogenen amerikanische AMPP-Studie (amerikanische Migräne-Prävalenz und Prevention-Studie) mit 5 Jahres Follow-Up untersuchte nun den Zusammenhang zwischen Medikamenten-Übergebrauch und dem Übergang von einer episodischer Migräne zu chronischem Kopfschmerz [22]. Analysiert wurden hierzu die Daten von 9031 Patienten. 55\% der Patienten nutzen zur Akuttherapie nichtsteroidale Antirheumatika, $2 \%$ entwickelten einen chronischen Kopfschmerz. Bei den 20\% der Teilnehmer, die Triptane nutzten, kam es bei $3 \%$ pro Jahr zu einem Übergang zu einem chronischen Verlauf. Die Analyse demonstrierte zudem, dass bei Individuen mit weniger als $10 \mathrm{Kopfschmerztagen/Monat} \mathrm{die}$ Einnahme von NSARs im Gegensatz zur Population mit 10-14 Einnahmetagen kein erhöhtes Risiko für eine Chronifizierung birgt. Bei den Triptanen hingegen zeigte sich ein fast linearer $\mathrm{Zu}$ sammenhang zwischen Einnahmetagen und Chronifizierungsrate. Die Studie bestätigt somit die deutschen Vordaten zur Entstehung eines MÜKS und unterstreicht, dass es insbesondere bei Patienten mit Triptaneinnahme auf eine Reglementierung der Einnahmetage zu achten ist.

Zur Therapie des Übergebrauchkopfschmerzes hat sich in der klinischen Praxis die Kombination aus Medikamentenpause und prophylaktische Therapie bewährt. Einige Patienten tun sich mit der Entzugsbehandlung auch ohne auf den ersten Blick erkennbares Risikoprofil diesbezüglich deutlich schwerer als andere und haben eine schlechtere Prognose. 2 im letzten Jahr veröffentlichte Arbeiten beschäftigten sich mit diesem Problem im Kontext genetischer Varianten und konnten Hinweise für einen möglichen genetischen Zusammenhang finden [23,24]. Dies ist ein neuer und prinzipiell interessanter Ansatz, allerdings scheint eine Praxisrelevanz noch in der ferneren Zukunft zu liegen.

Anhand eines Konsensus-Protokolls einer internationalen Kopfschmerzexperten-Gruppe wurde in einer multizentrischen multinationalen, allerdings nur unkontrollierten Studie mit 376 Patienten in 4 europäischen und 2 südamerikanischen Studien- zentren die Therapie von Patienten mit MÜKS evaluiert [25]. Die Mehrheit der Patienten wurde mit einem ambulanten Detoxifikations-Programm behandelt, anschließend erfolgte ein 6-monatiger Nachbeobachtungszeitraum. Sowohl bezüglich der Kopfschmerztage als auch bezüglich der Medikamenteneinnahme konnte ein gutes Ansprechen der Patienten für den vollständigen Beobachtungszeitraum gezeigt werden. Zum Abschluss der Studie betrieben zwei Drittel der Patienten anhaltend keinen Übergerbrauch mehr, bei 46,5\% konnte der Kopfschmerz nach Anwendung des Protokolls zu insofern reduziert werden, dass nur noch ein episodischer Kopfschmerz bestand. Sowohl die ambulante als auch stationäre Versorgung zeigten sich effektiv. Eine sehr interessante Studie aus Skandinavien demonstrierte erneut, wie effektiv auch einfache Maßnahmen zur Bekämpfung des MÜKS sein können. In dieser doppelblinden, cluster-randomisierten und parallel-kontrollierten Studie wurde Wirksamkeit einer Kurzintervention zur Optimierung der Entzugsbehandlung in der Primärversorgung untersucht [26]. Hierzu wurden, nach Screening der Patienten bezüglich eines $\mathrm{MOH}$ Gruppen an Hausärzten randomisiert. 23 der Hausärzte erhielten im Rahmen einerCME-Fortbildung ein Kurzinterventions(KI)Training, während bei 27 Hausärzten dagegen nichts an der Behandlungstrategie geändert wurde („Business as usual“). Im Rahmen der KI wurden Patienten über den Zusammenhang zwischen dem Übergebrauch und chronischem Kopfschmerz informiert. Des Weiteren wurde auf dem Boden der erhobenen „Severity of Dependence“-Skala auch Feedback zum Individuellen Risiko gegeben und allgemeine wie explizite Informationen und Anweisungen zum Entzug/zur Reduktion vermittelt. Es wurde Wert darauf gelegt, dass der Patient aktiv die Entscheidung fällt den Übergebrauch zu beenden. Beide primären Endpunkte (Reduktion der Einnahme- und Kopfschmerztag 3 Monate nach der Intervention) wurden durch einen verblindeten Untersucher bei den 60 Patienten erhoben. Es zeigte sich eine deutliche Überlegenheit in der KI-Gruppe ( $p<0,001$, adjustierte Reduktion der Kopfschmerz- und Medikationstage um 7,3 bzw. 7,9 Tage/Monat in der KI-Gruppe im Vergleich anderen Gruppe). Bei 50\% der Patienten konnte so eine „Dechronifizierung“ der Kopfschmerzen erreicht werden. In der nicht interventionsgruppe wurde dieses nur bei $6 \%$ der Patienten möglich. Die Studie unterstreicht wie wichtig und effektiv es ist, gerade im primären Sektor MÜKS zu berücksichtigen und zu behandeln, zumal hier auch oftmals der initiale Patientenkontakt stattfindet und frühzeitig eine weitere Chronifizierung von Kopfschmerzen verhindert werden könnte.

\section{Kopfschmerzen vom Spannungstyp}

Episodische Kopfschmerzen vom Spannungstyp (EKST) werden von weiten Teilen der Bevölkerung berichtet. Bei einem kleinen Teil der Patienten einwickelt sich aus den episodisch auftretenden Kopfschmerzen ein chronischer Verlauf (CKST). Die Pathophysiologie dieser häufigen Erkrankung ist nicht genau bekannt. Es scheinen pro-inflammatorische Mechanismen beteiligt $\mathrm{zu}$ sein. Bei KST-Patienten war im Vergleich zu Kontrollen Interleukin-8 im Serum erhöht. Bei der Untergruppe Patienten, die während der Untersuchung an Kopfschmerzen litten, war zudem das Monozyten-Chemoattraktor-Protein-1 erhöht [27]. Auch die Plasma-Tryptamin-Spiegel liegen bei Patienten mit CKST höher, wodurch die Modulation von eingehenden nozizeptiven Inputs gestört sein könnte [28]. Patienten mit CKST haben zudem eine erhöhte perikraniale Druckdolenz [29]. Erwachsene Patienten 
mit kombinierter Migräne und KST, aber auch mit alleinigem KST oder Migräne haben gehäuft Nackenschmerzen $(89,3,88,4$ und 76,2\%) [30]. Dieser Befund konnte für KST in einer Gruppe Jugendlicher nicht bestätigt werden [31]. Patienten mit KST leiden häufiger unter einer Beeinträchtigung des Schlafes i.S. von Tagesmüdigkeit, reduzierter subjektiver Schlafqualität sowie verändertem Schlafmuster bei normaler Schlafdauer. Die Autoren schlussfolgerten, dass KST-Patienten mehr Schlaf als gesunde Menschen benötigen [32]. Eine Meta-Analyse zu Kopfschmerzen und Adipositas weist auf einen möglichen Zusammenhang zwischen Adipositas und KST, insbesondere bei chronischem Verlauf hin [33]. Auch hormonelle Faktoren scheinen das Auftreten eines KST zu beeinflussen, wenn dieser Zusammenhang auch geringer ist als bei der Migräne. In einer brasilianischen Studie wurde in einer Gruppe von 422 Studentinnen (18-45 Jahre) die Häufigkeit von menstruellen Kopfschmerzen erhoben. 31,8\% litten unter menstruationsassoziierten Kopfschmerzen, davon die meisten unter einer Migräne (27,5\%) und nur ein Zehntel (3,1\%) unter einem KST [34].

Insgesamt liegen trotz der Häufigkeit der Erkrankung nur wenige Daten über die akute Behandlung vor. In der Akuttherapie zeigt sich in einer Meta-Analyse von 4 randomisierten, doppelblinden, Plazebo-kontrollierten, crossover Studien eine fixe Kombination aus ASS, Paracetamol und Koffein wirksamer (kein Kopfschmerz 2 Stunden nach Einnahme bei 28,5\%, „number needed to treat (NNT)“ 9,5) als die Einnahme von Paracetamol (21,0\%) oder Plazebo (18,0\%) [35]. Eine Meta-Analyse aller randomisierter, doppelblinder Studien, die eine Substanz gegen Plazebo oder eine andere Substanz getestet haben, zeigte ähnliche Wirkstärken für Paracetamol, Ibuprofen und Ketoprofen. Die NNT für Schmerzfreiheit nach 2 Stunden liegt für Paracetamol $1000 \mathrm{mg}$ bei 8,7 , für Ibuprofen $400 \mathrm{mg}$ bei 8,9 und für Ketoprofen $25 \mathrm{mg}$ bei 9,8 [36]. Für die parenterale Akuttherapie zeigten Metamizol (NNT 4), Chlorpromazin (NNT 4) und Metoclopramid (NNT 2) eine dem Plazebo überlegene Wirkung [37].

Studien im Bereich der medikamentösen Prophylaxe wurden auch im letzten Jahr nicht durchgeführt. Die trizyklischen Antidepressiva und hier vor allem Amitriptylin gelten unverändert als Therapeutika der ersten Wahl [38].

Bei der nicht-medikamentösen prophylaktischen Therapie konnten randomisierte Plazebo-kontrollierte Studien Effekte der manuellen Therapie in der Reduktion der Kopfschmerzhäufigkeit nachweisen $[39,40]$. Es zeigte sich bei 44 Patienten mit häufigem episodischen KST ein anhaltender Effekt über 1 und 3 Monate (Reduktion der Kopfschmerzhäufigkeit von 40 bzw. $50 \%)$ nach manueller Therapie im Vergleich zu einer ShamBehandlung [41].

\section{New Daily Persistent Headache}

$\nabla$

Der neu aufgetretene tägliche Kopfschmerz (New Daily Persistent Headache, NDPH) ist ein seltenes Krankheitsbild, das akut auftritt, bereits nach 24 Stunden persistiert und über mindestens 3 Monate anhält [42]. Nach den neuen ICHD-III beta-Kriterien kann die Symptomatik sowohl einem Kopfschmerz vom Spannungstyp als auch einer Migräne entsprechen. Der Verlauf ist oft langwierig und die Behandlung schwierig [43]. Remittierende Verläufe werden jedoch beobachtet. Häufig treten begleitend Angst- und depressive Störungen auf $[44,45]$. Die medikamentöse Therapie entspricht der Prophylaxe beim Kopfschmerz vom Spannungstyp oder der Migräne. Prospektive Studien liegen nicht vor und die Ansprechrate in den vorliegenden Studien ist sehr niedrig [46].

\section{Idiopathische intrakranielle Hypertension (IIH, Pseudotumor cerebri)}

Symptome der IIH sind Kopfschmerzen, Sehstörungen und Gesichtsfeldeinschränkungen, Stauungspapillen und pulsatiler Tinnitus. Die Erkrankung tritt vor allem bei übergewichtigen Frauen im gebärfähigen Alter auf. In der Liquorpunktion findet sich ein erhöhter Liquoreröffnungsdruck von $\geq 250 \mathrm{~mm}$ in seitlicher Lagerung. Therapeutisch sollte dringend eine Gewichtsreduktion angestrebt werden. Zudem werden Topiramat, Acetazolamid und Furosemid bislang trotz unzureichender Studienlage eingesetzt. Eine multizentrische randomisierte, Plazebo-kontrollierte verblindete Studie an 38 Zentren in den Vereinigten Staaten untersuchte zwischen 2010 und 2012 bei 165 Patienten (vorwiegend Frauen) die Wirkung von Actazolamid. Verabreicht wurde die maximal verträgliche Dosis (Zieldosis $4 \mathrm{~g} / \mathrm{Tag}$, mittlere erreichte Dosis 2,5 g/Tag) oder Plazebo über 6 Monate. Zudem wurde in beiden Gruppen ein Gewichtsreduktionsprogramm durchgeführt. Der primäre Endpunkt war eine Änderung der mittleren Abweichung in der Perimetrie zwischen Einschluss in der Studie und nach 6 Monaten. Sekundäre Endpunkte waren u.a. die Intensität der Kopfschmerzen, das Ausmaß der Stauungspapille und das Körpergewicht. In der Verum-Gruppe zeigte sich eine statistisch signifikante Verbesserung des Gesichtsfeldes, des Ausmaßes der Stauungspapille und eine deutlichere Gewichtsreduktion (7,5 vs. 3,45 kg). Die Kopfschmerzen wurden jedoch nicht signifikant beeinflusst. Nach 6 Monaten berichteten $69 \%$ der Verum- und 68\% der Plazebo-Gruppe über Kopfschmerzen [47]. In einer weiteren Untersuchung besserten sich die Kopfschmerzen bei $43 \%$ von 44 nach üblichen Standards (Acetazolamid und/oder Topiramat) behandelten Patienten innerhalb von 12 Monaten, meist innerhalb der ersten Wochen nach Diagnosestellung, deutlich [48].

\section{Trigeminusneuralgie}

$\nabla$

Die Therapie der Trigeminusneuralgie bleibt weiterhin eine große Herausforderung allerdings wird zunehmend an neuen medikamentösen Therapieoptionen gearbeitet. Ein neuer, selektiver Natriumkanalblocker der Nav 1,7 Untereinheit zeigte in einer kürzlich vorgestellten Phase-2-Studie eine gute Wirksamkeit und exzellente Verträglichkeit. Die Studie testete CNV10 14802150 mg 3× täglich bei 67 Patienten mit klassischer Trigeminusneuralgie und zeigte nach 28 Tagen eine 60\%ige Reduktion der Trigeminusneuralgieattacken im Vergleich zu lediglich $12 \%$ unter Plazebo. Die Schmerzintensität verminderte sich um 55\% unter CNV1014802 und 18\% unter Plazebo. Im Durchschnitt wurde eine Reduktion auf einer numerischen rating Skala (NRS) von 2,3 Punkten erreicht (Convergence pharmaceuticals press release 2014).

Breits 2012 führten Wu et al. die erste kontrollierte, klinische Studie zur Wirksamkeit von Botulinumtoxin Typ A zu Behandlung der Trigeminusneuralgie bei 42 Patienten durch und konnten eine signifikante Schmerzreduktion um über 50\% der behandelten Patienten nach 12 Wochen feststellen [49]. Insgesamt $75 \mathrm{U}$ wurden in den schmerzhaften Bereich subkutan oder intramucosal injiziert. Nebenwirkungen durch die Behandlung 
wurden nicht berichtet. Eine aktuelle Studie konnte ein ähnliches Ergebnis an 84 Patienten mit Trigeminusneuralgie zeigen (Plazebo N=28, BTX-A 25 U N = 27, BTX-A 75 U N = 29) [50]. Hierbei waren $25 \mathrm{U}$ Botox genauso effektiv wie $75 \mathrm{U}$ und die Wirksamkeit setzte bereits nach einer Woche ein und hielt über die gesamte Beobachtungszeit von 8 Wochen an. Leider haben beide Studien methodische Fehler was ihre Aussagekraft einschränkt und eine generelle Empfehlung zum Einsatz von Botulinumtoxin zur Behandlung der TN noch nicht ausgesprochen werden kann. Eine dritte kontrollierte Studie läuft derzeit in Deutschland (BoTN-Studie) und wird zeigen ob Botulinumtoxin in der nahen Zukunft eine vernünftige Therapieoption der Trigeminusneuralgie ist.

Neuromodulationsverfahren werden auch bei Trigeminusneuralgie zunehmend eingesetzt. Eine aktuelle Studie untersuchte die Wirksamkeit der transkraniellen Gleichstromstimulation (tDCS) des Motorkortex bei 10 Patienten mit therapierefraktärer Trigeminusneuralgie [51]. Die Stimulation erfolgte täglich über 14 Tage und die Patienten konnten das Stimulationsgerät nach individueller Anpassung und Einstellung mit nach Hause nehmen. Es zeigte sich eine signifikante Reduktion der Schmerzintensität um 29\% ( $p=0,0008)$, bei ähnlich guter Reduktion der Attackenfrequenz. Dieser Vergleich verfehlte aber die statistische Signifikanz im Vergleich zur Scheinstimulation (Sham). Ein größeres Patientenkollektiv muss in weiterführenden, multizentrischen Studien untersucht werden um die tDCS als neue Therapieoption der Trigeminusneuralgie zu bestätigen.

\section{Trigemino-autonome Syndrome}

$\nabla$

Bisher gab es keine eindeutige Definition des chronisch therapierefraktären Clusterkopfschmerzes. Die aktuelle Kopfschmerzklassifikation (ICDH-III beta) sieht lediglich eine Definition des chronisches Clusterkopfschmerzes vor, wobei Clusterpatienten hierfür für ein Jahr Clusterattacken aufweisen müssen, ohne dass es zu Pausen größer eines Monats kommt. Insbesondere für den Einsatz invasiverer Therapieverfahren (z.B. Neuromodulation) ist eine einheitliche Definition des chronischen therapierefraktären Clusterkopfschmerzes essentiell. Gleiches gilt für klinische Studien. Die Europäische Kopfschmerzgesellschaft (EHF) hat nun in Abstimmung mit internationalen Kopfschmerzexperten ein Konsensus-Paper bzgl. dieser Fragestellung veröffentlicht [52]. Als therapierefraktär gilt der chronische Clusterkopfschmerz demnach, wenn der Patient mindestens 3 schwere Clusterattacken pro Woche hat, die die Lebensqualität des Patienten wesentlich beeinflussen. Zudem müssen 3 prophylaktische Therapien, für die sich in Plazebo-kontrollierten Studien ein Wirksamkeitsbeleg findet, in maximaler Dosierung über einen ausreichenden Zeitraum ohne ausreichenden Therapieerfolg eingenommen worden sein. Diese neue Definition wird hoffentlich durch eine präzisere Identifikation dieser Patientengruppe zu einer Verbesserung der heute oftmals noch unzureichenden Therapie der Patienten mit chronischem therapierefraktären Clusterkopfschmerz führen.

Die klare circadiane und circannuale Rhythmik der Clusterattacken wurde immer als Beleg für die maßgebliche Beteiligung des Hypothalamus in der Pathophysiologie dieses Krankheitsbildes angesehen. Diese schien auch durch eine initiale Bildgebungsstudie belegt [53], die eine Zunahme an grauer Substanz im Hypothalamus zeigte. Neuere umfangreiche Bildgebungsstudien zeigen nun, dass sich vielfältige Veränderungen in zahlreichen Strukturen des Gehirns finden, sodass eher von einer Fehlfunktion eines gesamten Netzwerkes als von einer monokausalen Strukturstörung im Hypothalamus auszugehen ist $[54,55]$. Sicherlich spielt aber der Hypothalamus als Hauptrelaystation der Schmerzverarbeitung auch in der Pathophysiologie des Clusterkopfschmerzes eine wichtige Rolle.

Die Wichtigkeit des Hypothalamus zeigt sich auch in neuesten Liquoruntersuchungen. Bei Patienten mit episodischem und chronischen Clusterkopfschmerz zeigte sich verglichen mit gesunden Kontrollen eine niedrigere Konzentration von Hypocretin 1 im Liquor [56]. Dieses Neuropeptid wird im Hypothalamus gebildet und ist maßgeblich u.a. an der Regulation des Schlafrhythmus beteiligt. Möglichweise weist Orexin A auch zusätzliche antinozizeptive Effekte auf und nimmt auf diesem Weg Einfluss auf die Pathophysiologie des Clusterkopfschmerzes. Eine Beteiligung an der Entstehung der Narkolepsie ist bereits gut belegt. In Metaanalysen zeigte sich zudem eine genetisch bedingte Veränderung des Hypokretin Rezeptor 2 bei Patienten mit Clusterkopfschmerz. Allerdings ließ sich dieser Zusammenhang in einer großen genetischen Assoziationsstudie bei 575 Clusterpatienten nicht nachweisen [57], sodass die Studienlage diesbezüglich weiterhin unklar bleibt.

\section{Interessenkonflikt}

M. Obermann hat Reiseunterstützung und Referentenhonorare von Biogen Idec, Genzyme, Novartis, Sanofi-Aventis, Pfizer, Teva und Heel bekommen. Wissenschaftliche Projekte wurden von Allergan, Electrocore, Heel und dem Bundesministerium für Bildung und Forschung finanziell unterstützt.

D. Holle wurde von Allergan und Grünenthal unterstützt. HansChristoph Diener hat Honorare für die Planung, Ausführung oder Teilnahme an Klinischen Studien, Teilnahme an Advisory Boards oder Vorträge erhalten von: Addex Pharma, Alder, Allergan, Almirall, Amgen, AstraZeneca, Autonomic Technology, Bayer Vital, Berlin Chemie, Boehringer Ingelheim, Bristol-Myers Squibb, Chordate, CoLucid, Coherex, Electrocore, GlaxoSmithKline, Grünenthal, Janssen-Cilag, Labrys Biologicals, Lilly, La Roche, 3M Medica, Menerini, Minster, MSD, Novartis, Johnson \& Johnson, Pierre Fabre, Pfizer, Schaper und Brümmer, Sanofi, St. Jude Medical, TEVA und Weber \& Weber. Finanzielle Unterstützung für Forschungsprojekte wurde gewährt von: Allergan, Almirall, AstraZeneca, Bayer, Electrocore, GSK, Janssen-Cilag, MSD und Pfizer. Kopfschmerzforschung an der Universitätsklinik für Neurologie und dem Westdeutschen Kopfschmerzzentrum Essen erfolgt durch: Deutsche Forschungegemeinschaft (DFG), Bundesministerium für Bildung und Forschung (BMBF), und die Europäische Union (EU). Prof. Hans-Christoph Diener besitzt keine Aktien oder Anteile von Pharmafirmen.

K. Solbach, S. Nägel und J. Burmeister geben an dass kein Interessenkonflikt besteht.

\footnotetext{
Literatur

1 Burch RC, Loder S, Loder $E$ et al. The prevalence and burden of migraine and severe headache in the United States: updated statistics from government health surveillance studies. Headache 2015; 55: 21-34

2 Schramm SH, Moebus S, Lehmann $N$ et al. The association between stress and headache: A longitudinal population-based study. Cephalalgia 2014; 34: 986-993
} 
3 Bruffaerts R, Demyttenaere K, Kessler RC et al. The Associations Between Preexisting Mental Disorders and Subsequent Onset of Chronic Headaches: A Worldwide Epidemiologic Perspective. J Pain Off 2015; 16: $42-52$

4 Blaauw BA, Dyb G, Hagen $K$ et al. The relationship of anxiety, depression and behavioral problems with recurrent headache in late adolescence - a Young-HUNT follow-up study. J Headache Pain 2015; 16: 10

5 Rist PM, Buring JE, Kurth T. Dietary patterns according to headache and migraine status: A cross-sectional study. Cephalalgia 2014; epub ahead of print

6 Buettner C, Burstein R.. Association of statin use and risk for severe headache or migraine by serum vitamin D status: A cross-sectional population-based study. Cephalalgia 2014; epub ahead of print

7 Lipton RB, Fanning KM, Serrano $D$ et al. Ineffective acute treatment of episodic migraine is associated with new-onset chronic migraine. Neurology 2015; 84: 688-695

8 Wells RE, Markowitz SY, Baron EP et al. Identifying the Factors Underlying Discontinuation of Triptans. Headache J Head Face Pain 2014; 54: 278-289

9 Goadsby PJ, Grosberg BM, Mauskop A et al. Effect of noninvasive vagus nerve stimulation on acute migraine: An open-label pilot study. Cephalalgia 2014; 34: 986-993

10 Fujita $M$, Sato $K$, Nishioka $H$ et al. Oral sumatriptan for migraine in children and adolescents: A randomized, multicenter, placebo-controlled, parallel group study. Cephalalgia 2014; 34: 365-375

11 Evers $S$. The efficacy of triptans in childhood and adolescence migraine. Curr Pain Headache Rep 2013; 17: 342

12 Tronvik E, Stovner LJ, Helde G et al. Prophylactic treatment of migraine with an angiotensin II receptor blocker: a randomized controlled trial. JAMA 2003; 289: 65-69

13 Stovner LJ, Linde M, Gravdahl GB et al. A comparative study of candesartan versus propranolol for migraine prophylaxis: A randomised, triple-blind, placebo-controlled, double cross-over study. Cephalalgia 2013; 34: 523-532

14 Villalón CM, Olesen J. The role of CGRP in the pathophysiology of migraine and efficacy of CGRP receptor antagonists as acute antimigraine drugs. Pharmacol Ther 2009; 124: 309-323

15 Ho TW, Connor KM, Zhang Y et al. Randomized controlled trial of the CGRP receptor antagonist telcagepant for migraine prevention. Neurology 2014; 83: 958-966

16 Dodick DW, Goadsby PJ, Silberstein SD et al. Safety and efficacy of ALD403, an antibody to calcitonin gene-related peptide, for the prevention of frequent episodic migraine: a randomised, double-blind, placebo-controlled, exploratory phase 2 trial. Lancet Neurol 2014; 13 : $1100-1107$

17 Dodick DW, Goadsby PJ, Spierings ELH et al. Safety and efficacy of LY2951742, a monoclonal antibody to calcitonin gene-related peptide, for the prevention of migraine: a phase 2, randomised, double-blind, placebo-controlled study. Lancet Neurol 2014; 13: 885-892

18 Bigal ME, Escandon R, Bronson $M$ et al. Safety and tolerability of LBR101 , a humanized monoclonal antibody that blocks the binding of CGRP to its receptor: Results of the Phase 1 program. Cephalalgia 2013; 34: 483-492

19 Dilli E, Halker R, Vargas B et al. Occipital nerve block for the short-term preventive treatment of migraine: A randomized, double-blinded, placebo-controlled study. Cephalalgia 2014; epub ahead of print

20 Conforto $A B$, Amaro E, Gonçalves $A L$ et al. Randomized, proof-ofprinciple clinical trial of active transcranial magnetic stimulation in chronic migraine. Cephalalgia 2014; 34: 464-472

21 Bendtsen L, Munksgaard S, Tassorelli $C$ et al. Disability, anxiety and depression associated with medication-overuse headache can be considerably reduced by detoxification and prophylactic treatment. Results from a multicentre, multinational study (COMOESTAS project). Cephalalgia 2014; 34: 426-433

22 Lipton RB, Serrano D, Nicholson RA et al. Impact of NSAID and Triptan use on developing chronic migraine: results from the American Migraine Prevalence and Prevention (AMPP) study. Headache 2013; 53: $1548-1563$

23 Cargnin S, Viana $M$, Ghiotto $N$ et al. Functional polymorphisms in COMT and SLC6A4 genes influence the prognosis of patients with medication overuse headache after withdrawal therapy. Eur J Neurol 2014; 21: 989-995

24 Cargnin S, Viana M, Sances $G$ et al. Combined effect of common gene variants on response to drug withdrawal therapy in medication overuse headache. Eur J Clin Pharmacol 2014; 70: 1195-1202

25 Tassorelli $C$, Jensen $R$, Allena $M$ et al. A consensus protocol for the management of medication-overuse headache: Evaluation in a multicentric, multinational study. Cephalalgia 2014; 34: 645-655
26 Kristoffersen ES, Straand J, Vetvik KG et al. Brief intervention for medication-overuse headache in primary care. The BIMOH study: a double-blind pragmatic cluster randomised parallel controlled trial. J Neurol Neurosurg Psychiatry 2015; 86: 505-512

27 Domingues RB, Duarte H, Rocha NP et al. Increased serum levels of interleukin-8 in patients with tension-type headache. Cephalalgia 2014; epub ahead of print

28 D'Andrea G, D'Amico D, Bussone $G$ et al. Tryptamine levels are low in plasma of chronic migraine and chronic tension-type headache. Neurol Sci 2014; 35: 1941-1945

29 Aaseth $K$, Grande RB, Lundqvist C et al. Pericranial tenderness in chronic tension-type headache: the Akershus population-based study of chronic headache. J Headache Pain 2014; 15: 58

30 Ashina S, Bendtsen L, Lyngberg AC et al. Prevalence of neck pain in migraine and tension-type headache: A population study. Cephalalgia 2015; 35: 211-219

31 Blaschek A, Decke S, Albers L et al. Self-reported neck pain is associated with migraine but not with tension-type headache in adolescents. Cephalalgia 2014; 34: 895-903

32 Engstrøm $M$, Hagen $K$, Bjørk $M$ et al. Sleep quality, arousal and pain thresholds in tension-type headache: a blinded controlled polysomnographic study. Cephalalgia 2014; 34: 455-463

33 Chai NC, Scher AI, Moghekar A et al. Obesity and headache: part I - a systematic review of the epidemiology of obesity and headache. Headache 2014; 54: 219-234

34 Melhado EM, Bigal ME, Galego AR et al. Headache classification and aspects of reproductive life in young women. Arq Neuropsiquiatr 2014; 72: 17-23

35 Diener H-C, Gold M, Hagen M. Use of a fixed combination of acetylsalicylic acid, acetaminophen and caffeine compared with acetaminophen alone in episodic tension-type headache: meta-analysis of four randomized, double-blind, placebo-controlled, crossover studies. J Headache Pain 2014; 15: 76

36 Moore RA, Derry S, Wiffen PJ et al. Evidence for efficacy of acute treatment of episodic tension-type headache: Methodological critique of randomised trials for oral treatments. Pain 2014; 155: 2220-2228

37 Weinman $D$, Nicastro $O$, Akala $O$ et al. Parenteral treatment of episodic tension-type headache: a systematic review. Headache 2014; 54: $260-268$

38 Barbanti P, Egeo G, Aurilia C et al. Treatment of tension-type headache: from old myths to modern concepts. Neurol Sci 2014; 35 (Suppl 1): $17-21$

39 Espí-López GV, Rodríguez-Blanco C, Oliva-Pascual-Vaca A et al. Effect of manual therapy techniques on headache disability in patients with tension-type headache. Randomized controlled trial. Eur J Phys Rehabil Med 2014; 50: 641-647

40 Gemma VE-L, Antonia G-C. Efficacy of manual and manipulative therapy in the perception of pain and cervical motion in patients with tension-type headache: a randomized, controlled clinical trial. J Chiropr Med 2014; 13: 4-13

41 Rolle G, Tremolizzo L, Somalvico F et al. Pilot trial of osteopathic manipulative therapy for patients with frequent episodic tension-type headache. J Am Osteopath Assoc 2014; 114: 678-685

42 Headache Classification Committee of the International Headache Society (IHS). The International Classification of Headache Disorders, $3^{\text {rd }}$ edition (beta version). Cephalalgia 2013; 33: 629-808

43 Rossi P, Tassorelli C, Allena $M$ et al. Focus on therapy: hemicrania continua and new daily persistent headache. J Headache Pain 2010; 11: 259-265

44 Peres MFP, Lucchetti G, Mercante JPP et al. New daily persistent headache and panic disorder. Cephalalgia Int J Headache 2011; 31: 250-253

45 Robbins MS. New daily-persistent headache and anxiety. Cephalalgia 2011; 31: 875-876

46 Takase Y, Nakano M, Tatsumi $C$ et al. Clinical features, effectiveness of drug-based treatment, and prognosis of new daily persistent headache (NDPH): 30 cases in Japan. Cephalalgia 2004; 24: 955-959

47 NORDIC Idiopathic Intracranial Hypertension Study Group Writing Committee.Wall M, McDermott MP et al. Effect of acetazolamide on visual function in patients with idiopathic intracranial hypertension and mild visual loss: the idiopathic intracranial hypertension treatment trial. JAMA 2014; 311: 1641-1651

48 Yri HM, Rönnbäck C, Wegener $M$ et al. The course of headache in idiopathic intracranial hypertension: a 12-month prospective follow-up study. Eur J Neurol 2014; 21: 1458-1464

$49 \mathrm{Wu}$ C-J, Lian Y-J, Zheng Y-K et al. Botulinum toxin type A for the treatment of trigeminal neuralgia: results from a randomized, doubleblind, placebo-controlled trial. Cephalalgia 2012; 32: 443-450 
50 Zhang H, Lian Y, Ma Y et al. Two doses of botulinum toxin type A for the treatment of trigeminal neuralgia: observation of therapeutic effect from a randomized, double-blind, placebo-controlled trial. J Headache Pain 2014; 15: 65

51 Hagenacker $T$, Bude $V$, Naegel $S$ et al. Patient-conducted anodal transcranial direct current stimulation of the motor cortex alleviates pain in trigeminal neuralgia. J Headache Pain 2014; 15: 78

52 Mitsikostas DD, Edvinsson L, Jensen RH et al. Refractory chronic cluster headache: a consensus statement on clinical definition from the European Headache Federation. J Headache Pain 2014; 15: 79

53 May A, Ashburner J, Büchel C et al. Correlation between structural and functional changes in brain in an idiopathic headache syndrome. Nat Med 1999; 5: 837
54 Naegel S, Holle D, Obermann M. Structural Imaging in Cluster Headache. Curr Pain Headache Rep 2014; 18: 1-8

55 Naegel S, Holle D, Desmarattes N et al. Cortical plasticity in episodic and chronic cluster headache. NeuroImage Clin 2014; 6: 415-423

56 Barloese $M$, Jennum $P$, Lund $N$ et al. Reduced CSF hypocretin-1 levels are associated with cluster headache. Cephalalgia 2014; epub ahead of print

57 Weller CM, Wilbrink LA, Houwing-Duistermaat IJ et al. Cluster headache and the hypocretin receptor 2 reconsidered: A genetic association study and meta-analysis. Cephalalgia 2014; epub ahead of print 